\title{
The relationship between CrossFit performance and grip strength
}

\author{
(D)Emily Haynes, (iD Mark DeBeliso \\ Southern Utah University, Department of Kinesiology and Outdoor Recreation, Cedar City, UT, USA
}

\begin{abstract}
There is a growing interest in maximizing CrossFit (CF) performance as the sport becomes more economically viable at elite levels. The ability to delineate the physiological demands of the sport of CF allows coaches and athletes to develop more efficacious exercise programming in order to maximize the athlete's potential for success at the most elite and lucrative levels of competition. There is also a growing interest in increasing health and fitness in the general population as obesity and chronic disease rates continue to rise. Hand grip strength (HGS) is an indicator of total body strength, mortality, morbidity and independence among aging adults. Given that CF is the "Sport of Fitness", it would be of interest to determine the relationship between HGS and CF performance. The current study examined the relationship between CF performance and hand grip strength (HGS). It was hypothesized that $\mathrm{CF}$ performance would have a meaningful significant relationship with HGS. Fifteen $(\mathrm{n}=15)$ female CF participants (age 30.9 \pm 7.1 years, height $160.3 \pm 4.8 \mathrm{~cm}$, body mass $64.5 \pm 9.6 \mathrm{~kg}$ ) of varying experience levels (51.9 \pm 30.6 months) were assessed for HGS and CF performance measures. The CF performance measures were assessed via a Workout of the Day (WOD) comprised of 3 rounds of 30 seconds at each of the following stations: fan bike (FB) for maximum calories, air squats (AS) for maximum repetitions, sit-ups (SU) for maximum repetitions, and burpees (BP) for maximum repetitions. Each 30 second work interval was followed by 2 minutes and 30 seconds of rest to ensure full recovery of the phosphagen energy system. Scores were reported as the mean number of repetitions completed across the 3 attempts at each movement station. Individual
\end{abstract}

movements as well as total repetitions were then compared to HGS with Pearson correlation coefficients (r). Sit-up performance demonstrated a positive moderately $(\mathrm{r}=0.44)$ significant relationship with mean HGS $(\mathrm{p}<0.05)$. Neither total WOD performance nor any other individual movement had a significant relationship with HGS $(p>0.05)$. Within the parameters of this study, CF participants exhibited a moderate relationship between HGS and sit-up scores.

Keywords. CrossFit, dynamometer, grip strength.

\section{Introduction}

CrossFit $^{\mathrm{TM}}$ (CF) was founded in 2000 by Greg Glassman in Santa Cruz, California and its popularity has been increasing exponentially since. There are now over 13,000 affiliate gyms located across 142 different countries (Beers, 2014). CF defines itself as constantly varied functional movements performed with high intensity over broad time and modal domains (CrossFit Inc., 2017a). Some practitioners use CF in order to increase general physical preparedness and overall health and fitness. Others practice $\mathrm{CF}$ as a performance sport and attend a growing number of $\mathrm{CF}$ competitions (CrossFit Inc., 2017b). As the competition side of CF grows, more lucrative endorsement deals and prize purses are becoming available, continuing to spur rapid growth and increased involvement. The male and female

Received: January 21, 2019 - Accepted: February 20, 2019 - Published: March 30, 2019

To cite this article: Haynes E, DeBeliso M. The relationship between CrossFit performance and grip strength. Turk J Kinesiol, 2019; 5(1): 15-21. 
winners of the Reebok CrossFit Games ${ }^{\mathrm{TM}}$ each take home \$300,000 (The Reebok CrossFit Games Competition Rulebook, 2018), and Reebok offers a minimum of another $\$ 400,000$ in endorsements amongst Games athletes of their choosing (Pyfferoen, 2018). Likewise, Nike and other brands have also started to endorse CF athletes (Guelde, 2014), as such, becoming an elite CF athlete has become a viable full time job.

Despite the burgeoning business of competitive $\mathrm{CF}$, research is still scarce regarding the physiological demands of the sport. Crossfit aims to improve fitness across 10 domains: cardiovascular and respiratory endurance, stamina, strength, flexibility, power, speed, coordination, agility, balance, and accuracy (Glassman, 2002). Comparisons between traditional resistance training (RT) methods and CF were initially favorable, with CF ranking equally or more efficacious for developing strength and power (Barfield, Channell, Pugh, Tuck, Pendel, 2012; Barfield \& Anderson, 2014; De Sousa et al, 2016; Fernández-Fernández et al., 2015). However, a recent meta-analysis (Claudino et al., 2018) determined that of the 10 fitness domains that CF claims to address, only 5 have been researched: cardiovascular and respiratory endurance, stamina, strength, flexibility, and power (Eather et al., 2016; Murawska-Cialowicz et al., 2015). The remaining 5 domains (speed, coordination, agility, balance, and accuracy) have yet to be examined.

Several studies have examined body composition (BC) changes due to $\mathrm{CF}$, presumably as a measure of health, with mixed results. Healthy but sedentary participants showed no significant changes in BC after 8 weeks of CF (Heinrich, 2014), but sedentary cancer survivors showed significant decreases in fat mass and relative body fat as well as significant increases in lean body mass after only 5 weeks of CF training (Heinrich, 2015). Participants who were already physically active had significant changes in lean body mass after 12 weeks of CF training, but only females showed a significant decrease in body mass index (BMI) and relative body fat (Murawska, 2014). Female teenagers also showed significant decreases in BMI with 8 weeks of CF Teens training, but their male counterparts did not (Eather, 2015). Finally, a recent meta-analysis of CF literature to has found no significant relationship between $\mathrm{CF}$ training and any assessment of BC. The metaanalysis also determined that only $6 \%$ of included CF studies had a high level of evidence at a low risk of bias (Claudino, 2018).

In the sport of CrossFit, it follows that competitions are a test of the $10 \mathrm{CF}$ domains (cardiovascular and respiratory endurance, stamina, strength, flexibility, power, speed, coordination, agility, balance, and accuracy) (CrossFit Inc., 2017). Researchers have determined relationships between exogenous characteristics and performance in many other sports, but the CF field is relatively unexplored. Crossfit is, however, unique in that it is comprised of several other sports - an elite CF athlete must be a proficient weightlifter, powerlifter, and gymnast, as well as a sprinter and an endurance athlete. Notably, researchers have found significant relationships between hand grip strength (HGS) and performance amongst weightlifters, raw powerlifters, and gymnasts. Fry et al. (2006) determined that $84 \%$ of American junior male weightlifters could be successfully predicted to be elite or non-elite based on body mass index (BMI), vertical jump, relative fat, HGS, and torso angle during an overhead squat (2006). Schoffstall et al. (2010) found that HGS of raw powerlifters correlated significantly with their squat $(\mathrm{r}=0.95)$, bench press $(\mathrm{r}=0.98)$, deadlift $(\mathrm{r}=0.97)$, and total $(r=0.97)$. Powerlifters were also found to have significantly greater HGS than gymnasts, who, in turn, had significantly greater HGS than nonexercisers (Ruprai et al., 2016). Regarding sport performance, HGS has been documented to be a covariant of lean muscle mass, sprinting and jumping ability, and training experience (Cronin, 2017), all of which are likely related to CF performance. As such, it would seem reasonable to 
suspect that there is a meaningful relationship between CF performance and HGS.

Hand grip strength may also be of particular interest to CF practitioners with goals of basic health and fitness, rather than performance. Though HGS was used in only one of the existing CrossFit studies (Meier et al., 2015), HGS is widely used as a predictive measure of several health markers. The Honolulu Heart Program (Rantanen et al., 1999) began in 1965 with 6089 Japanese-American healthy men, then aged 45 to 68 years. At the initiation of the program maximal HGS was assessed. By the 19911993 follow period, 2259 men had died. Of the remaining survivors, 3218 men participated in the reassessment of HGS. Greater decrements in HGS were associated with greater functional limitations over the interceding 25 years. Men who tested in the lowest third of HGS upon re-testing were at more than 2 times the risk of self-care disability than those in the highest third of HGS. In other words, HGS in men aged 45 to 68 years is highly predictive of their ability to independently care for themselves 25 years later.

Other studies have indicated that HGS is a good predictor of total body strength and functional ability (DeBeliso et al., 2015a; DeBeliso et al., 2015b), total muscle strength (Wind et al., 2010), mortality (Granic et al., 2017), morbidity (Norman et al., 2011), and cognition (Praetorious et al., 2016) in aging adults. Among participants at least 85 years old, a decline in HGS over the subsequent 10 years was indicative of a $16 \%$ greater risk of mortality in men and a 33\% greater risk in women. Those that improved their HGS decreased their risk of mortality by $31 \%$ (Granic et al., 2017). In elderly pneumonia patients admitted to a hospital, HGS was highly predictive of death and/or readmission within 1 year of discharge (Bohannon et al., 2004). In participants who completed baseline testing at 80 years of age and re-testing at 2 year intervals thereafter, decrements in HGS occurred conjointly with decrements in cognition. The closer to death the participants became, the closer the association between the two measures (Praetorious et al., 2016). Among male and female participants over the age of 50 , those with one chronic disease had significantly lower HGS values than those without a chronic condition, and those suffering from multi-morbidity had significantly lower values than those with only one disease (Yorke et al., 2015).

As elderly populations struggle with frailty, loss of independence, morbidity, and decreased quality of life, the likelihood of decline in these areas is accurately associated with diminished HGS. It would be worthwhile to investigate which particular exercise protocols are most efficacious in improving HGS as a marker of overall health and fitness. Certain programs have already demonstrated the ability to improve HGS. A 30 day yoga camp significantly improved HGS in both male and female adults. Ten days of yoga was enough to significantly improve the HGS of children, and 15 days significantly improved HGS in patients with rheumatoid arthritis (Dash, 2001). In college-aged adults, right hand HGS improved significantly after 5 weeks of High Intensity Interval Training (HIIT) with kettlebells and battle ropes. Notably, left hand HGS showed no significant changes (Meier, 2015). However, a study that compared light physical exercise with a health education program for 72- to 84-year-olds found no improvement in HGS related to either treatment (Santanasto, 2017), indicating that perhaps a certain level of intensity or frequency of exercise is required in order to improve HGS and, accordingly, the associated health benefits.

Further research to determine the parameters of exercise type, intensity, frequency, and volume necessary to elicit measurable improvements in HGS aging and general populations would appear warranted. Given the growing popularity of $\mathrm{CF}$, its geographical availability, and its demonstrated high levels of community, satisfaction, and motivation (Claudino et al., 2018), CF has the potential to be an excellent program choice for those seeking to improve overall health and fitness. It would be of interest to examine the relationship between $\mathrm{CF}$ and 
HGS, as an association between the two would indicate that CF may lead to measurable improvements in health that would support the preservation of independence and full faculties into senior years. As such, the purpose of the current study is to examine the relationship between HGS and $\mathrm{CF}$ performance.

\section{Methods}

\section{Participants}

A convenience sample of 15 female CrossFit athletes over the age of 18 were recruited from CrossFit 215, which is housed within Requisite Fitness in Philadelphia, Pennsylvania. All participants were healthy, non-pregnant women with 3 to 96 months of CrossFit experience. Participants were free of any neuromuscular, orthopedic, or neurological conditions that might interfere with physical activity. They were recruited via an email sent out to the entire membership body, calling for volunteers.

Prior to any testing or assessment, permission from the Institutional Review Board was obtained. Each participant was provided with a written informed consent form to read and sign before they were included in the study. It was made abundantly clear to all participants that participation was strictly voluntary and could be withdrawn at any time for any reason.

\section{Instruments and apparatus}

Hand grip strength was measured using a Camry hydraulic hand grip dynamometer (EH101; Camry, Guangdong Province, China) (range 0-90 kg; accuracy $0.1 \mathrm{~kg}$ ) (Latorre Román, 2017), loaned from Subversus Fitness in Philadelphia, PA. Participants took part in a specially programmed $\mathrm{CF}$

Workout of the Day (WOD) using Assault AirBike fan bikes, a Rogue AB-2 ab-mat, and bodyweight movements.

Five separate workout stations were set up with screens between each so that participants could not see each other. Each station measured a minimum of 1.8 meters by 1.8 meters and consisted of one fan bike, one ab-mat, and ample space to complete the required bodyweight movements. Due to the potential confusion around actual time at which to work or rest, each station was equipped with its own timing sheet, instructing the participant which movement to execute at which time on the clock. The workout was timed using a Muscle Driver USA programmable gym timer (No Limits; MDUSA, South Carolina, USA).

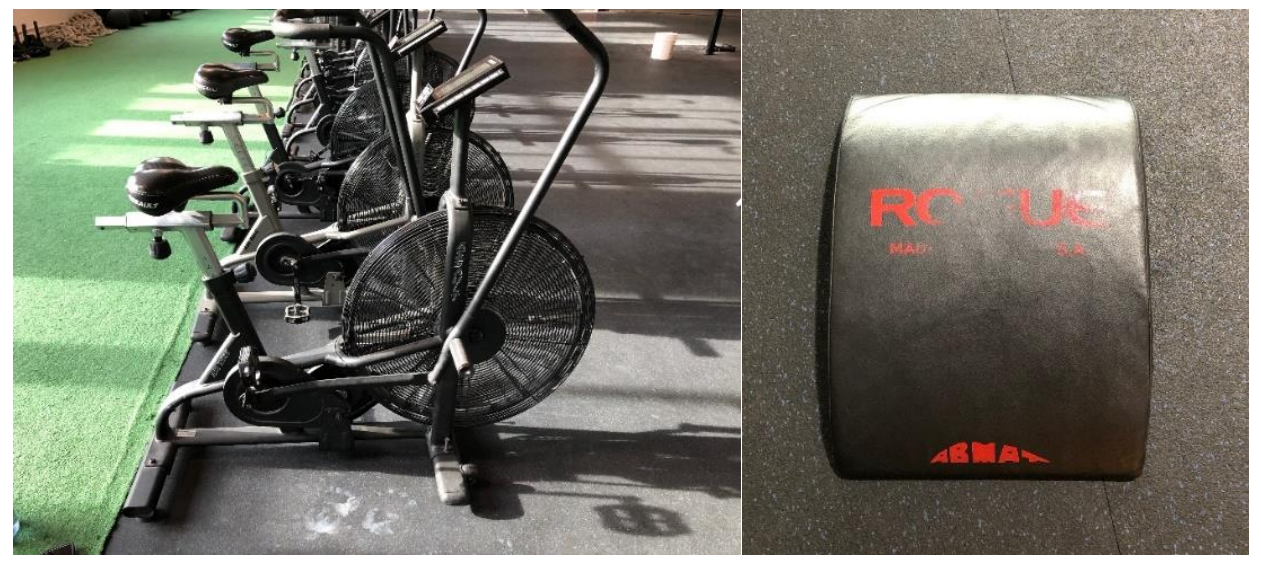

Figure 1. Assault AirBike and Rogue ab-mat for sit-ups at the Requisite Fitness in Philadelphia, Pennsylvania, US. 


\section{Procedures}

The study was administered in three (3) separate one (1) hour increments, and participants chose which hour to attend based on preference and availability. There were 2 sessions on November 3 at 9 a.m. and 10 a.m., and one session on November 6 at 4:30 p.m. It has been demonstrated that HGS does not vary throughout the day (Patel, 2004, Young et. al, 1989). Upon arrival, participants were individually taken to a private room where their height, weight, dominant hand, age, and number of months of CF experience were recorded. Participants were measured without shoes or excessive clothing.

The Camry dynamometer was then used to assess HGS using the guidelines set forth by the American Society of Hand Therapists: the shoulder was adducted and neutrally rotated, with the elbow at $90^{\circ}$ flexion and a neutral forearm. Participants were allowed to self-select wrist position to allow for maximal gripping (Fess, 1981). The standard instructions given were as follows: "squeeze the gauge as hard as you possibly can while maintaining this standard position." Subjects completed their trials on their dominant hand, then rested for 1 minute before repeating for a total of 3 trials. Each HGS trail lasted 3 seconds. A running stopwatch was visible to both the administrator and participant at all times. Dynamometer readings were recorded by hand and later transferred to an electronic database. The intraclass reliability coefficient (ICC) for the three trials of HGS scores was calculated as ICC $=0.89$.

Upon completion of HGS testing, all participants were brought to the main part of the facility as a group for a dynamic warm-up. The dynamic warmup consisted of two rounds of one minute on the fan bike, ten Spiderman steps, ten scapula push-ups, and ten air squats. In each hour increment, there were five (5) participants. The WOD was then explained to the group as follows:

"You will complete three (3) rounds of thirty (30) seconds of work at each of four stations, with two and a half (2.5) minutes of rest between each station. The four stations will be fan bike, air squats, sit-ups, and burpees. Complete as many repetitions at each station as you can. The fan bike will be measured in calories. Do not concern yourself with counting your repetitions - I will be counting for you. I will not count out loud".

Each movement was demonstrated for the participants before they were given time to ask clarifying questions. Air squat standards required participants to break parallel at the bottom and fully extend their hips at the top. Sit-up standards required participants to lower their upper bodies to the floor, touching the floor with their hands above their heads, then sit-up to a point at which the shoulders crossed the vertical line of the hips. Burpee standards required participants to touch their chest to the floor then jump and clap with arms fully extended overhead. No standards were given regarding the fan bike. Due to previous CF experience, all participants were familiar with the movements and standards.

Each participant was led to their randomly assigned workout station. Participants were not told their scores at any point during the workout. The working period at each station began with the administrator instructing "begin." At thirty (30) seconds in, the administrator instructed the participant to rest. The two minute and thirty second rest periods were included to ensure full recovery of the ATP-CP pathway after its dominant role in the work period (Baechle \& Earle, 2008; Martinopoulou et al., 2011). Each station completed the workout in a staggered fashion, so that participants were working while the others were resting at their respective stations. This was done solely in the name of expediency. Participants were allowed to bring water to their station and hydrate at their discretion. Upon completion of the WOD, participants were informed that they had permission to use any space or equipment in the gym in order to cool down for the subsequent fifteen minutes. Participants were reminded not to speak with each other or any incoming new participants about any part of the 
testing. All WOD data was recorded by hand and later transferred to an electronic database.

The WOD was designed in a manner to assess movements that did not involve the manipulation of implements in attempt to separate CF performance in general from barbell or gymnastics-specific performance.

\section{Design and analysis}

The variables assessed in this study included: HGS (kgs), fan bike (calories), air squats (repetitions), situps (repetitions), and burpees (repetitions). The association between HGS and the four exercise making up the WOD (fan bike, air squats, sit-ups, and burpees) were conducted with Pearson correlation coefficients (r). Individual HGS scores were also compared to normative values as set forth by Wang et al. (2018). Significance for the study was set a priori $\alpha \leq 0.05$. All statistical analyses were completed in MS Excel 2013. The data analysis spread sheet was peer reviewed for accuracy as described by Al Tarawneh \& Thorne (2017).

\section{Results}

Fifteen female participants (right-handed=14, lefthanded=1) completed the study without incident and the demographics are presented in Table 1. The average age, height, body mass, and CF experience were: $30.9 \pm 7.1$ years, $160.3 \pm 4.8 \mathrm{~cm}, 64.5 \pm 9.6 \mathrm{~kg}$, and $51.9 \pm 30.6$ months respectively. The results of the Pearson correlation coefficients ( $r$ ) suggested a moderately significant relationship between HGS and sit-up performance $(\mathrm{p}<0.05)$ (see Table 5). No significant relationships were found between HGS and total performance, fan bike performance, air squat performance, or burpee performance $(\mathrm{p}>0.05)$. The average fan bike performance was $9.9 \pm 2.3$ calories in 30 seconds. The average air squat performance was $27.3 \pm 3.4$ repetitions. The average sit-up performance was $16.5 \pm 2.5$ repetitions. The average burpee performance was $11.5 \pm 1.8$ repetitions. Ten (10) participants scored at or above their normative reference value for HGS, presented in Table 4. The remaining 5 scored below.

Table 1

Participant descriptive information (Mean \pm SD).

\begin{tabular}{lcccc}
\hline & Age (years) & Height $(\mathrm{cm})$ & Mass $(\mathrm{kg})$ & Experience (months) \\
\hline Female $(\mathrm{n}=15)$ & $30.9 \pm 7.1$ & $160.3 \pm 4.8$ & $64.5 \pm 9.6$ & $51.9 \pm 30.6$ \\
\hline
\end{tabular}

Table 2

Workout of the day (WOD) scores.

\begin{tabular}{|c|c|c|c|c|c|}
\hline & $\begin{array}{l}\text { Fan Bike } \\
\text { Calories }\end{array}$ & $\begin{array}{l}\text { Air Squats } \\
\text { Repetitions }\end{array}$ & $\begin{array}{c}\text { Sit-ups } \\
\text { Repetitions }\end{array}$ & $\begin{array}{c}\text { Burpees } \\
\text { Repetitions }\end{array}$ & Total Score \\
\hline Female $(n=15)$ & $9.9 \pm 2.3$ & $27.3 \pm 3.4$ & $16.5 \pm 2.5$ & $11.5 \pm 1.8$ & $65.2 \pm 7.9$ \\
\hline
\end{tabular}

Average across three trials; Mean \pm SD

Table 3

HGS trial data $(\mathrm{kg})$.

\begin{tabular}{lccccc}
\hline & Trial 1 & Trial 2 & Trial 3 & Trials Total & Trials Mean \\
\hline Female $(\mathrm{n}=15)$ & $28.6 \pm 4.5$ & $30.1 \pm 5.4$ & $30.3 \pm 5.6$ & $89.1 \pm 14.6$ & $29.7 \pm 4.9$ \\
\hline
\end{tabular}

Average across three trials; Mean \pm SD 


\section{Table 4}

Measured HGS compared to reference values and experience.

\begin{tabular}{lcccc}
\hline Participant & Age & Mean HGS $(\mathrm{kg})$ & $\begin{array}{c}\text { Normative Percentile } \\
\text { Range (Actual) }\end{array}$ & $\begin{array}{c}\text { CF Experience } \\
\text { (months) }\end{array}$ \\
\hline 1 & 31 & 29.3 & $25-<50$ & 48 \\
2 & 45 & 31.4 & $50-<75$ & 60 \\
3 & 43 & 28.9 & $25-<50$ & 72 \\
4 & 32 & 19.0 & $0-<10$ & 3 \\
5 & 32 & 31.0 & $50-<75$ & 96 \\
6 & 31 & 32.4 & $50-<75$ & 90 \\
7 & 31 & 31.9 & $50-<75$ & 96 \\
8 & 25 & 25.9 & $25-<50$ & 60 \\
9 & 40 & 34.7 & $75-<90$ & 48 \\
10 & 33 & 20.1 & $0-<10$ & 72 \\
11 & 27 & 36.2 & $75-<90$ & 36 \\
12 & 25 & 27.7 & $25-<50$ & 3 \\
13 & 18 & 36.0 & $75-<90$ & 30 \\
14 & 26 & 32.0 & $50-<75$ & 60 \\
15 & 24 & 28.8 & $50-<75$ & 5 \\
\hline
\end{tabular}

Table 5

Summary of comparison of total performance and mean HGS.

\begin{tabular}{lccc}
\hline Performance Measure & $\begin{array}{c}\text { HGS } \\
r\end{array}$ & $\begin{array}{c}\text { Significant } \\
\text { at } \mathrm{P}<0.05\end{array}$ & Size \\
\hline Total fan bike calories & 0.43 & No & \\
Total air squats & 0.15 & No & \\
Total sit-ups & 0.44 & Yes & Moderate \\
Total burpees & -0.01 & No & \\
Total all & 0.34 & No & \\
\hline
\end{tabular}

Normative reference percentiles were obtained from Table 1 (Wang et al., 2018) and are based on sex and age. For example, Participant 1's HGS measures between the $25^{\text {th }}$ and $50^{\text {th }}$ percentile of all $30-34$ year old women in the United States. Highlighted rows indicate HGS below the $50^{\text {th }}$ percentile of normative reference values.

\section{Discussion}

The purpose of this study was to determine if a meaningful relationship existed between $\mathrm{CF}$ performance and HGS. It was hypothesized that CF performance would have a significant meaningful relationship with HGS. The study results demonstrated that sit-up performance had a moderately significant relationship with HGS. However, neither total performance nor the performance scores of any other movements had a significant relationship with HGS. The significant correlation between HGS and sit-ups indicated that there may be a relationship between HGS and core strength, though further research is needed to 
determine if the correlation is movement-specific or if it can be extrapolated more generally to abdominal strength or endurance. Overall, the data refute the existence of a meaningful relationship between $\mathrm{CF}$ performance and HGS.

The majority of participants in the current study (60\%) demonstrated HGS above normative reference 50th percentiles (Wang et al., 2018). Two of the participants had approximately 3 months of $\mathrm{CF}$ experience. If these two participants were removed from the data set, $77 \%$ of the CF participants in the current study would have demonstrated HGS above normative reference 50th percentiles (Wang et al., 2018). Several studies have established correlations between HGS and barbell athletes as well as gymnastics athletes (Ruprai et al., 2016; Schoffstall et al., 2010; Fry et al., 2006). High HGS values could be expected from this population because of the significant amount of time spent manipulating barbells and other weighted implements in their regular CF training, as well as time spent manipulating their own bodyweight around bars and rings.

The current study specifically chose to assess movements that did not involve the manipulation of objects in order to separate $\mathrm{CF}$ performance in general from barbell- or gymnastics-specific performance. Participants with higher HGS scores could already be presumed to perform better on a test like 30 seconds of max effort pull-ups or deadlifts as repeated coupling with a pull-up or Olympic bar should contribute to the development of HGS. Bodyweight movements and the minimal amount of hand-gripping required for the fan bike were deliberately chosen for the current investigation to determine if HGS meaningfully correlated with the general physical fitness required for $\mathrm{CF}$ performance. Another factor in our decision was the varied experience level of participants - we did not want to risk injury by asking participants to perform 30 seconds of moderate-weight deadlifts, for example. Though this may be done in a CF class, it would be under a coach's direct supervision and guidance. We were of the opinion that researchers should not attempt to wear multiple hats at once - we chose movements that would release us from the need for movement correction and allow us to simply observe the study without undue concern for safety. A more challenging study that employed a WOD where participants were performing more complex exercises would certainly be feasible with either more experienced athletes or more research staff. Because the movements that we chose to measure did not involve gripping objects, the performance variables became more of a measure of anaerobic fitness, aerobic fitness, and bodyweight exercise proficiency.

Previous research has demonstrated that elite and sub-elite performers can be accurately stratified using multiple variables to include HGS and anaerobic capacity in a variety of field sports (James et al., 2016) and combat sports (Franchini et al., 2005; Guidetti et al., 2002; Nikooie et al., 2017). However, to our knowledge no direct relationship between HGS and anaerobic fitness has yet been established. It is therefore not surprising that no significant relationships were determined between HGS and fan bike, air squat, burpee, or total performance. Though there is little research regarding HGS and aerobic endurance, it is generally theorized that endurance athletes would not exhibit high HGS because of that population's generally low body mass. Larger body mass often correlates with higher HGS (Cronin, 2017).

HGS may be generally related to performance of core-to-extremity movements that utilize the kinetic chain, building momentum from the core out toward the hand(s) (Cronin, 2017). Examples of this type of movement include throwing an object or swinging a bat or racket. Because momentum begins at the core for those movements, it therefore follows that high performance requires core strength. The significant relationship between sit-ups and HGS may confirm this correlation between core strength and HGS. This theory has yet to be fully explored or developed, but 
this study indicates that perhaps future research on the topic is warranted.

Within the parameters of this study, HGS proved unrelated to anaerobic fitness or non-gripping bodyweight movements, with the exception of situps. We found it surprising that no relationships (other than sit-ups) were found between HGS and CF performance. Upon further consideration, it is possible that relationships remained insignificant due to lack of diversity in performance measures and/or inclusion of covariates that could not be controlled for, such as height, weight, age, and training experience (Wang, 2018; Cronin, 2017; Rantanen, 1999; Wadsworth, 1992). A larger, more varied sample size might allow researchers to control those variables. It is possible that a correlation between HGS and performance of the CF variables assessed in the current study might emerge if these aforementioned variables could be controlled for.

The comparatively high HGS scores of the current study participants were likely predictable as they are consistent with previous research regarding gymnasts and barbell athletes (Fry et al., 2006; Schoffstall et al., 2010; Ruprai). This may suggest that CF is likely a good system for improving HGS, and therefore useful for general health and fitness. It supports the claim by CrossFit that CF participation benefits aging populations and helps them to stay healthy and independent. Regarding the sport of CF, it is difficult to compare this study with previous studies of other sports. CF is, on the whole, somewhat difficult to study simply because it is an amalgamation of so many other sports and activities. In order to determine the importance of HGS to $\mathrm{CF}$ elite performance, it would be necessary to measure HGS in a sample of CF elite-level athletes, as well as the CF sub-elite. It is worth noting that the one participant that could potentially be classified as an elite-level competitor (5 Regionals appearances, including 3 top-10 finishes) included in this study presented HGS above her normative value, but only in the $70^{\text {th }}$ percentile of normative values and 73rd percentile of the current study participants.
Obviously more research would be necessary to determine if HGS is correlated with success in CF as it is in certain other sports.

Overall, the above 50 percentile norm HGS scores of $60 \%$ of the participants supports the theory that occupations and hobbies involving manual manipulation of implements correlates with higher HGS. Further, as mentioned above, if the participants with only 3 months of CF experience were removed from the data set, a total of $77 \%$ of the participants would exhibit above 50 percentile norm HGS. The sit-up data also supports the less-developed theory that HGS is related to core-to-extremity proficiency. This is particularly interesting because CrossFit Inc. claims core-to-extremity movement as a defining characteristic of their program. It is the foundational core-to-extremity principle upon which the use of compound movements and the gymnastics kip is based. The significant correlation between sit-up performance and HGS revealed in the current study indicates that this principle may be sound.

The most obvious limitation of the current study is the small sample size. Due to the limited number of participants, there was a tremendous variety of height, weight, and experience level. Other sportspecific studies have determined that these characteristics are covariates of HGS (Wang, 2018; Cronin, 2017; Rantanen, 1999; Wadsworth, 1992). If a sample size could be obtained with more homogenous height, weight, and experience levels, correlations between performance and HGS might prove meaningful and significant. Additionally, the workout might not have been challenging enough. Only 3 of 15 participants had less than 6 months of CF experience. Since the majority of participants were fairly experienced $\mathrm{CF}$ athletes, most were capable of completing a similar number of repetitions in each 30 second interval. Because the WOD was designed to provide adequate rest, those repetitions were also repeatable over all 3 rounds. A more challenging workout might have provided a greater strata of scores, which in turn might identify 
stronger correlations between total WOD performance and HGS.

Ideally, future research would rectify these issues in order to provide a broader understanding of the physiological needs for success in the sport of fitness known as CrossFit. Future research might also help to determine which implements most improve HGS as $\mathrm{CF}$ includes a multitude of implements beyond barbells and rings to include kettlebells, dumbbells, ropes, and odd objects. For those pursuing $\mathrm{CF}$ as means for general health and fitness, a longitudinal study in which CF practitioners could be tracked into their senior years in order to determine their rates of independence and mortality would be very useful.

If $\mathrm{CF}$ is to be used for general fitness as the hedge against senescence that it claims to be (Glassman, 2017), then it seems likely that it must include implement work - i.e. barbells, pull-up bars, or gymnastics rings. Current literature does indicate that consistently gripping and manipulating implements likely correlates to improved HGS, and that improved HGS correlates, whole body strength, functional ability, longevity, independence, and decreases in morbidity (Cronin, 2017; Rantanen et al., 1999; DeBeliso et al., 2015a; DeBeliso et al, 2015b; Granic et al., 2017; Norman et al., 2011). With regard to elite CF performance, it appears that additional HGS training may not be necessary. In order to qualify for the CF Games, potential competitors require a requisite amount of physical strength, aerobic and anaerobic capacity. Further, a potential competitor should be proficiently skilled with: manipulating odd objects, the gymnastics rings, and performing the Olympic lifts. As such, it would be likely that the nature of $\mathrm{CF}$ training would indirectly lead to the requisite HGS levels required to compete at an elite CF level.

Within the parameters of this study, HGS is not correlated with overall $\mathrm{CF}$ performance, though there is a moderately significant correlation between HGS and sit-up performance. Participants with greater than 3 months $\mathrm{CF}$ experience tended to exhibit HGS above $50^{\text {th }}$ percentile normative levels, which indicates that $\mathrm{CF}$ may be a useful fitness regimen for aging populations. Future research should pursue the identification of better physiological predictors of CF performance.

\section{Conflict of Interest Declaration}

No funding was received for this research. The authors have no conflict of interest related to this research. This research has not been previously published.

\section{References}

AlTarawneh G, Thorne S. A pilot study exploring spreadsheet risk in scientific research. arXiv preprint arXiv:1703.09785. arvix.org, Ithca, NY, 2017.

Baechle TR, Earle RW. Essentials of strength training and conditioning. Champaign, IL: Human Kinetics, 2008.

Barfield JP, Anderson A. Effect of CrossFit on healthrelated physical fitness: A pilot study. Journal of Sport and Human Performance, 2014; 2(1): 23-28.

Barfield JP, Channell B, Pugh C, Tuck M, Pendel D. Format of basic instruction program resistance training classes: Effect on fitness change in college students. Physical Educator, 2012; 69(4): 325-341.

Beers E. Virtuosity goes viral. The CrossFit Journal, 2014; 6: 1-10.

Bellace JV, Healy D, Besser MP, Byron T, Hohman L. Validity of the Dexter Evaluation System's Jamar dynamometer attachment for assessment of hand grip strength in a normal population. Journal of Hand Therapy: Official Journal of the American Society of Hand Therapists, 2000; 13(1): 46-51.

Bohannon RW, Maljanian R, Ferullo J. Mortality and readmission of the elderly one year after hospitalization for pneumonia. Aging Clinical and Experimental Research, 2004; 16(1): 22-25.

Brown JT, Sobrero GL, Inman C, Stone W, Zagdsuren B, Arnett SW, Shafer MA, Lyons S, Maples JM, Crandall J, Callahan Z. Crossfit vs. circuit-trained individuals. Medicine Science in Sports and 
Exercise, $\quad 2015 ; \quad 47: \quad 800$. doi:10.1249/01.mss.0000478923.27125.12

Cadenas-Sanchez C, Sanchez-Delgado G, Martinez-Tellez B, Mora-Gonzalez J, Löf M, España-Romero V, Ruiz JR, Ortega FB. Reliability and validity of different models of TKK hand dynamometers. The American Journal of Occupational Therapy: Official Publication of the American Occupational Therapy Association, 2016; 70(4), 7004300010. doi:10.5014/ajot.2016.019117

Cronin J, Lawton T, Harris N, Kilding A, McMaster DT. A brief review of handgrip strength and sport performance. Journal of Strength and Conditioning Research, 2017; 31(11): 3187-3217. doi:10.1519/JSC.0000000000002149

CrossFit Inc. About the Games. Retrieved November 30, 2018, from https://games.crossfit.com/about-thegames, 2017b.

CrossFit Inc. Level 1 Training Guide (2nd ed.). Santa Cruz, California, 2017a.

Dash M, Telles S. Improvement in hand grip strength in normal volunteers and rheumatoid arthritis patients following yoga training. Indian Journal of Physiology and Pharmacology, 2001; 45(3): 355-360.

de Sousa AFM, dos Santos G, dos Reis T, Valerino A, Del Rosso S, Boullosa D. Differences in physical fitness between recreational CrossFit ${ }^{\circledR}$ and resistance trained individuals. Journal of Exercise Physiology Online, 2016; 19(5): 112-122.

DeBeliso M, Boham M, Harris C, Carson C, Berning JM, Sevene TG, Adams KJ. Grip and body strength measures in the mature adult: A brief report. International Journal of Science and Engineering Investigations, 2015a; 4(37): 83-86.

DeBeliso M, Boham M, Harris C, Carson C, Berning JM, Sevene TG, Adams KJ, Climstein M. Grip strength and functional measures in the mature adult: Brief report II. International Journal of Science and Engineering Investigations, 2015b; 4(39): 1-4.

Dunwoody L, Tittmar HG. Grip strength and inter-trial rest. Perceptual \& Motor Skills, 1996; 83(1): 275.

Eather N, Morgan P, Lubans D. Improving health-related fitness in adolescents: The CrossFit teens randomized controlled trial. Journal of Sports Sciences, 2015; 34(3): 1-15. doi:10.1016/j.jsams.2015.12.406

Fernández-Fernández J, Sabido-Solana R, Moya D, Sarabia JM, Moya M. Acute physiological responses during CrossFit workouts. European Journal of Human Movement, 2015; 35: 114-124.

Fess EE, Moran C. Clinical assessment recommendations. Indianapolis: American Society of Hand Therapists Monograph; 1981.

Franchini E, Takito M, Bertuzzi R. Morphological, physiological and technical variables in high-level college judoists. Science of Martial Arts, 2005; 1: 17.

Fry AC, Ciroslan D, Fry MD, LeRoux CD, Schilling BK, Chiu LZ. Anthropometric and performance variables discriminating elite American junior men weightlifters. Journal of Strength and Conditioning Research, 2006; 20(4): 861-866.

Glassman G. Foundations. The CrossFit Journal, 2002; 1: 1-8.

Glassman G. The world's most vexing problem. Lecture presented at CrossFit Level 1 Seminar in California, Aromas, September 3, 2017. Retrieved April 19, 2018, from https://journal.crossfit.com/article/cfjgreg-glassman-the-world-s-most-vexing-problem

Granic A, Davies K, Jagger C, Dodds RM, Kirkwood TL, Sayer AA. Initial level and rate of change in grip strength predict all-cause mortality in very old adults. Age and Ageing, 2017; 46(6): 970-976. doi:10.1093/ageing/afx087

Guelde L. Nike Signs Mat Fraser, Strengthens Elite Fitness Foothold, December 5, 2014. Retrieved October 21, 2018, from https://www.floelite.com/articles/5040021-nikesign-mat-fraser-strengthens-elite-fitness-foothold

Guidetti L, Musulin A, Baldari C. Physiological factors in middleweight boxing performance. Journal of Sports Medicine \& Physical Fitness, 2002; 42(3): 309-314.

Haidar SG, Kumar D, Bassi RS, Deshmukh SC. Average versus maximum grip strength: which is more 
consistent? Journal of Hand Surgery (Edinburgh, Scotland), 2004; 29(1): 82-84.

Hak PT, Hodzovic E, Hickey B. The nature and prevalence of injury during CrossFit training. Journal of Strength and Conditioning Research, Publish Ahead of Print, 2013.

Heinrich K, Becker C, Carlisle T, Gilmore K, Hauser J, Frye J, Harms C. High-intensity functional training improves functional movement and body composition among cancer survivors: A pilot study. European Journal of Cancer Care, 2015; 24(6): 812817. doi:10.1111/ecc.12338

Heinrich KM, Patel PM, O'Neal JL, Heinrich BS. Highintensity compared to moderate-intensity training for exercise initiation, enjoyment, adherence, and intentions: An intervention study. BMC Public Health, 2014; 14(1). doi:10.1186/1471-2458-14-789

James R, Thake C, Birch S. Relationships between measures of physical fitness change when agedependent bias is removed in a group of young male soccer players. Journal of Strength \& Conditioning Research, 2017; 31(8): 2100-2109.

Latorre Román PÁ, López DM, Aguayo BB, Fuentes AR, García-Pinillos F, Redondo MM. Handgrip strength is associated with anthropometrics variables and sex in preschool children: A cross sectional study providing reference values. Physical Therapy in Sport, 2017; 26: 1-6.

Martinopoulou K, Argeitaki P, Paradisis G, Katsikas C, Smirniotou A. The effects of resisted training using parachute on sprint performance. Biology of Exercise, 2011; 7(1): 7-23.

Mckenzie MJ. Crossfit improves measures of muscular strength and power in active young females. Medicine \& Science in Sports \& Exercise, 2015; 47: 797. doi:10.1249/01.mss.0000466164.99273.92

Meier J, Quednow J, Sedlak T. The effects of high intensity interval-based kettlebells and battle rope training on grip strength and body composition in collegeaged adults. International Journal of Exercise Science, 2015; 8(2): 124-133.

Murawska-Cialowicz E, Wojna J, Zuwala-Jagiello J. Crossfit training changes brain-derived neurotrophic factor and irisin levels at rest, after wingate and progressive tests, and improves aerobic capacity and body composition of young physically active men and women. Journal of Physiology and Pharmacology, 2015; 66(6): 811-821.

Nikooie R, Cheraghi M, Mohamadipour F. Physiological determinants of wrestling success in elite Iranian senior and junior Greco-Roman wrestlers. Journal of Sports Medicine and Physical Fitness, 2017; 57: 219-226.

Norman K, Stobäus N, Gonzalez MC, Schulzke J, Pirlich M. Hand grip strength: outcome predictor and marker of nutritional status. Clinical Nutrition (Edinburgh, Scotland), 2011; 30(2): 135-142. doi:10.1016/j.clnu.2010.09.010

Peolsson A, Hedlund R, Oberg B. Intra- and inter-tester reliability and reference values for hand strength. Journal of Rehabilitation Medicine, 2001; 33(1): 3641.

Praetorius Björk M, Johansson B, Hassing LB. I forgot when I lost my grip-strong associations between cognition and grip strength in level of performance and change across time in relation to impending death. Neurobiology of Aging, 2016; 38: 68-72. doi:10.1016/j.neurobiolaging.2015.11.010

Pyfferoen B. 10 Things you didn't know about the CrossFit-Reebok partnership. June 20, 2018. Retrieved October 21，2018， from http://thebarbellspin.com/functional-fitness/10things-you-didnt-know-about-the-crossfit-reebokpartnership/

Rantanen T, Guralnik JM, Foley D, Masaki K, Leveille S, Curb JD, White L. Midlife hand grip strength as a predictor of old age disability. Journal of the American Medical Association, 1999; 281(6): 558560.

Ruprai R, Tajpuriya S, Mishra N. Handgrip strength as determinant of upper body strength/physical fitness: A comparative study among individuals performing gymnastics (ring athletes) and gymnasium (powerlifters). International Journal of Medical Science and Public Health, 2016; 5(6): 1167. doi:10.5455/ijmsph.2016.09102015176

Santanasto AJ, Glynn NW, Lovato LC, Blair SN, Fielding RA, Gill TM, Guralnik JM, Hsu FC, King AC, 
Strotmeyer ES, Manini TM, Marsh AP, McDermott MM, Goodpaster BH, Pahor M, Newman AB. Effect of physical activity versus health education on physical function, grip strength and mobility. Journal of the American Geriatrics Society, 2017; 65(7): 1427-1433. doi:10.1111/jgs.14804

Schoffstall J, Morrison SD, Kozlik B, Boswell B. Grip strength and powerlifting performance. In: Southeastern Chapter of the American College of Sports Medicine Regional Conference, February 2010. Abstract retrieved from: https://www.researchgate.net/publication/2804088 78_Grip_Strength_and_Powerlifting_Performance

Serafini P, Hoffstetter W, Mimms H, Smith M, Kliszczewicz B, Feito Y. Body composition and strength changes following 16-weeks of highintensity functional training. Medicine \& Science in Sports \& Exercise, 2016; 48: 1001. doi:10.1249/01.mss.0000488009.97613.c7

Smith MM, Sommer AJ, Starkoff BE, Devor ST. Crossfitbased high-intensity power training improves maximal aerobic fitness and body composition. Journal of Strength And Conditioning Research, 2016; 27(11): 3159-3172. doi:10.1519/JSC.0b013e318289e59f

The Reebok CrossFit Games Competition Rulebook, no. 7, CrossFit, Inc., Santa Cruz, CA, USA, Jan 8 2018, p. 24. Accessed on: October 21, 2018 [Online]. Available: https://games.crossfit.com/rules/open

Timmons JF, Minnock D, Hone M, Cogan KE, Murphy JC, Egan B. Comparison of time-matched aerobic, resistance, or concurrent exercise training in older adults. Scandinavian Journal of Medicine \& Science in Sports, 2018; 28(11): 2272-2283.

Trossman P, Li P. The effect of the duration of inter-trial rest periods on isometric grip strength performance in young adults. Occupational Therapy Journal of Research, 1989; 9(6): 362-378.
Wadsworth C, Nielsen DH, Corcoran DS, Phillips CE, Sannes TL. Interrater reliability of hand-held dynamometry: effects of rater gender, body weight, and grip strength. The Journal of Orthopaedic and Sports Physical Therapy, 1992; 16(2): 74-81.

Wang YC, Sindhu B, Kapellusch J, Bohannon RW, Xiaoyan L. Hand-grip strength: Normative reference values and equations for individuals 18 to 85 years of age residing in the United States. Journal of Orthopaedic \& Sports Physical Therapy, 2018; 48(9): 685-693.

Weisenthal BM, Beck CA, Maloney MD, DeHaven KE, Giordano BD. Injury rate and patterns among CrossFit athletes. Orthopaedic Journal of Sports Medicine, 2014; 2(4): 2325967114531177. doi:10.1177/2325967114531177

Wind AE, Takken T, Helders PM, Engelbert RH. Is grip strength a predictor for total muscle strength in healthy children, adolescents, and young adults? European Journal of Pediatrics, 2010; 169(3): 281287. doi:10.1007/s00431-009-1010-4

Yorke AM, Curtis AB, Shoemaker M, Vangsnes E. Grip strength values stratified by age, gender, and chronic disease status in adults aged 50 years and older. Journal of Geriatric Physical Therapy, 2015; 38(3): 115-121.

Young VL, Pin P, Kraemer BA, Gould RB, Nemergut L, Pellowski M. Fluctuation in grip and pinch strength among normal subjects. The Journal of Hand Surgery, 1986; 14(1): 125-129.

Zagdsuren B, Evans GS, Inman C, Stone W, Arnett S, Schafer M, Lyons S, Maples JM, Crandall J, Callahan Z. Crossfit vs. circuit-training. Medicine \& Science in Sports \& Exercise, 2015; 47: 801. doi:10.1249/01.mss.0000478926.16823.b9 